\title{
SISTEMA SIMPLIFICADO DE DIREÇÃO ASSISTIDA PARA EMPILHADEIRAS E TRANSPALE- TEIRAS ELÉTRICAS
}

Victor Martins de Oliveira

Graduado em Engenharia Elétrica pelo Centro Universitário Augusto Motta - UNISUAM

$$
\text { - RJ - Brasil }
$$

victormartinsoliveira@yahoo.com.br

\section{Geraldo Motta Azevedo Júnior}

Doutor em Engenharia Elétrica pela COPPE/UFRJ - RJ - Brasil

Professor do Centro Universitário Augusto Motta - UNISUAM - RJ - Brasil

geraldomotta@unisuam.edu.br

\section{Antonio José Dias da Silva}

Mestre em Informática pela Universidade Federal do Rio de Janeiro - UFRJ - RJ - Brasil

Professor do Centro Universitário Augusto Motta - UNISUAM - RJ - Brasil

antoniojoseds@gmail.com

\section{Carlos Henriques Ventura do Rosário Oliveira}

Mestre em Sistemas de Gestão pela UFF - RJ - Brasil

Professor Adjunto da Universidade Federal Fluminense - UFF - RJ - Brasil

Professor do Centro Universitário Augusto Motta - UNISUAM - RJ - Brasil

choliveira@unisuam.edu.br 


\title{
RESUMO
}

O objetivo deste trabalho é propor um projeto, capaz de assistir eletricamente um sistema de direção para empilhadeiras e transpaleteiras elétricas, visando uma melhor eficiência e redução de custos operacionais. $O$ sistema eletrônico foi desenvolvido baseado em conceitos de eletrônica analógica e de potência para a realização de um controle bidirecional de um motor elétrico de ímãs permanentes. O funcionamento do sistema é demonstrado através de um protótipo, que foi desenvolvido com componentes atualmente utilizados em equipamentos embarcados em empilhadeiras e transpaleteiras elétricas. O sistema realiza o ajuste do ponto de acionamento, o ajuste de intensidade do acionamento do motor, o ajuste de fim de curso e possui uma proteção contra sobrecarga. Estes ajustes são realizados através de circuitos eletrônicos analógicos, que tiveram seu funcionamento comprovado através dos dados coletados durante o funcionamento do protótipo. Analisando o funcionamento de todo o sistema e verificando os resultados obtidos, concluiu-se que é possível e viável a implementação deste sistema em empilhadeiras e transpaleteiras elétricas.

Palavras-chave: Empilhadeiras. Transpaleteiras elétricas. Direção assistida. Sistema eletrônico.

\section{SIMPLIFIED ASSISTED STEERING SYSTEM FOR ELECTRIC FORKLIFT AND STACKERS}

\begin{abstract}
The objective of this work is to propose a project, capable of electrically assisting a steering system for electric forklifts and pallet trucks, aiming at a better efficiency and reduction of operational costs. The electronic system was developed based on concepts of analog electronics and power for the realization of a bidirectional control of an electric motor of permanent magnets. The functioning of the system is demonstrated through a prototype, which was developed with components currently used in equipment shipped on forklifts and electric pallet trucks. The system performs the adjustment of the activation point, the adjustment of the motor activation intensity, the limit switch adjustment and has an overload protection. These adjustments are made through analog electronic
\end{abstract}


circuits, which have been proven to work through data collected during the prototype's operation. Analyzing the operation of the entire system and verifying the results obtained, it was concluded that the implementation of this system in electric forklifts and pallet trucks is possible and viable.

KEY WORDS: Forklifts. Electric pallet trucks. Assisted steering. Electronic system.

\section{INTRODUÇÃO}

Empilhadeiras elétricas são definidas como equipamentos usados principalmente para carregar e descarregar mercadorias em paletes (estrados de madeira, metal ou plástico utilizados para a movimentação de cargas), sendo amplamente utilizados nos setores de logística, por proporcionarem uma eficiência significativa (RAMEP, 2017).

Essas máquinas utilizam sistemas de direção totalmente elétricos, ou seja, todo trabalho é realizado por um motor de direção, que é controlado por comandos elétricos e eletrônicos, o que traz uma facilidade para conduzi-las, porém quando em mau funcionamento as máquinas perdem seu controle de direção o que pode acarretar problemas, desde atrasos e paralisações em operações de carga e descarga, até acidentes envolvendo pessoas (REITER, 2016).

Neste contexto, o objetivo deste projeto é a implementação de um sistema de direção elétrica assistida capaz de proporcionar a mesma facilidade do sistema totalmente elétrico, porém de forma mais segura, confiável, com baixo custo e de fácil manutenção. Sabe-se que muitas vezes os sistemas totalmente elétricos tornam-se dispendiosos, por se tratar de sistemas importados que necessitam de uma manutenção de alta complexidade, o que os tornam muitas vezes inviáveis.

Além dos objetivos já citados espera-se ainda que o sistema proposto seja capaz de funcionar de forma que o mesmo não paralise a máquina em caso de defeito, dando condições mínimas ao operador de finalizar sua manobra e conduzi-la até o local de manutenção. 


\section{DESENVOLVIMENTO}

Para atingir os objetivos, o sistema foi dividido em três circuitos: acionamento, controle e potência. $O$ circuito de acionamento envia um sinal linear para o circuito de controle, que por sua vez também monitora o sinal enviado por um sensor de posição acoplado ao motor, que determina o fim de curso do sistema. Comparando essas informações com um sinal de PWM, o circuito de controle aciona o circuito de potência, responsável pelo controle bidirecional do motor.

\subsection{Circuito de Acionamento}

O sinal enviado pelo circuito de acionamento, é gerado através da manipulação do eixo de direção da máquina, que faz com que dois imãs inversamente polarizados, se movimentem e passem a influenciar o sensor de efeito hall UGN3503, através do campo magnético gerado por eles, o sensor está acoplado mecanicamente entre os dois imãs. Este sinal do sensor é amplificado através de um circuito amplificador não inversor, com o objetivo de se obter uma maior amplitude do sinal gerado. $O$ circuito de acionamento é alimentado por uma fonte de $12 \mathrm{~V}$, que está inserida no circuito de controle, e esse valor de $12 \mathrm{~V}$ é reduzido para $5 \mathrm{~V}$ com o objetivo de alimentar o UGN3503, conforme mostrado na Figura 1. 
Figura 1 - Circuito de acionamento

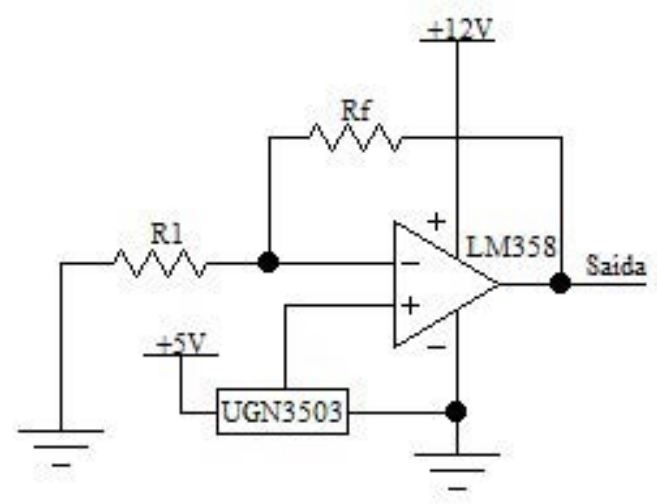

Fonte: (Autores).

O sensor de efeito hall está disposto mecanicamente de modo que a densidade do fluxo magnético dos imãs não atue no sensor enquanto a direção da máquina não seja acionada. Quando o operador acionar o eixo de direção da máquina, um dos imãs passa a influenciar magneticamente no sensor, que passa a variar seu sinal de saída de $2,5 \mathrm{~V}$ a $5 \mathrm{~V}$, quando acionado em um dos sentidos, e varia de 2,5V a $0 \mathrm{~V}$ quando o sistema é acionado no sentido oposto. Para facilitar a manipulação deste sinal no circuito de controle, o mesmo é amplificado de forma a variar de $6 \mathrm{~V}$ a $12 \mathrm{~V}$ em um dos sentidos, e de $6 \mathrm{~V}$ a $0 \mathrm{~V}$ no sentido oposto.

\subsection{Circuito de Controle}

O circuito de controle tem a função de condicionar os sinais enviados pelo circuito de acionamento e pelo sensor de posição, para fazer o correto acionamento do circuito de potência, responsável pelo controle bidirecional do motor. Através do sinal enviado pelo circuito de acionamento, o circuito de controle identifica o acionamento do eixo de direção determinando o ponto de acionamento do circuito, identifica também em que sentido ele está sendo acionado e se está dentro do ângulo de manobra. Com este mesmo sinal o circuito gera um sinal de PWM, que é enviado ao circuito de potência para fazer com que o motor seja acionado de forma proporcional ao acionamento 
do eixo de direção. Através de um circuito comparador, é identificado o acionamento do eixo de direção da máquina, comparando o sinal enviado pelo circuito de acionamento, com dois níveis de tensão ajustados por uma malha resistiva divisora de tensão. (Figura 2)

Figura 2 - Circuito que define o ponto de acionamento do sistema.

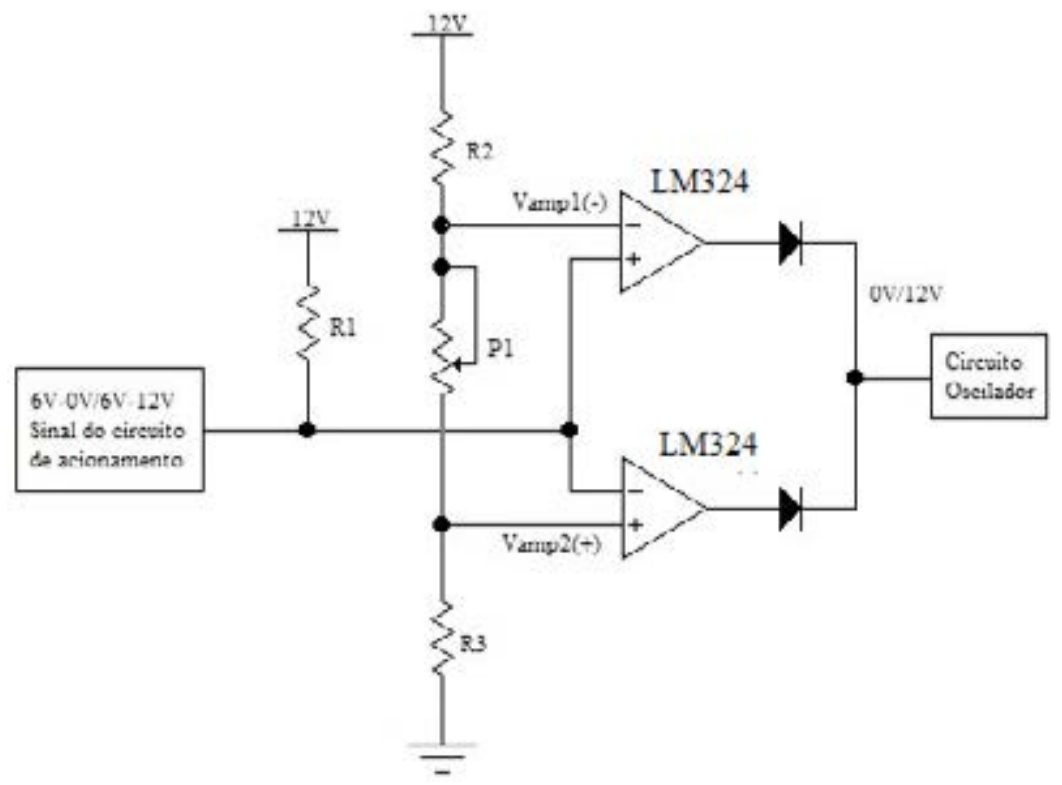

Fonte: (Autores).

Após a identificação do acionamento a saída do circuito muda de estado, de OV para $12 \mathrm{~V}$, ativando o circuito oscilador utilizado para gerar o sinal de PWM. Através do gerador de PWM, o circuito faz com que a partida do motor seja feita de forma controlada, e proporcional ao acionamento do eixo de direção. O sinal de PWM é gerado através da comparação do sinal enviado de um circuito oscilador de relaxação, com o sinal enviado pelo circuito de acionamento. O sinal enviado pelo circuito oscilador de relaxação é similar a um sinal de onda triangular, o oscilador entra em funcionamento após ser ativado pelo circuito do ponto de acionamento. A frequência do oscilador está dimensionada através do resistor $R_{13}$ e do capacitor $C_{1}$, com valores de $47 \mathrm{k} \Omega$ e $1 \mathrm{nf}$ respectivamente. Esses valores foram dimensionados para que fosse obtido o valor de 
frequência próximo a $15 \mathrm{kHz}$. O sinal do circuito de acionamento passa por dois circuitos amplificadores diferenciais, com o objetivo de fazer com que o sinal passe a variar de $4 \mathrm{~V}$ a $10 \mathrm{~V}$ quando o sistema é acionado em ambos os sentidos, estes valores são ajustados por $\mathrm{P}_{2}$ e $\mathrm{P}_{3}$. O Amplificador 3 está configurado como um amplificador diferencial e subtrai o sinal enviado pelo circuito de acionamento do valor ajustado pelo potenciômetro $\mathrm{P}_{2}$. O resultado obtido é multiplicado pelo ganho do circuito que é aproximadamente 2 , conforme mostrado na Figura 3.

Figura 3 - Circuito gerador de PWM

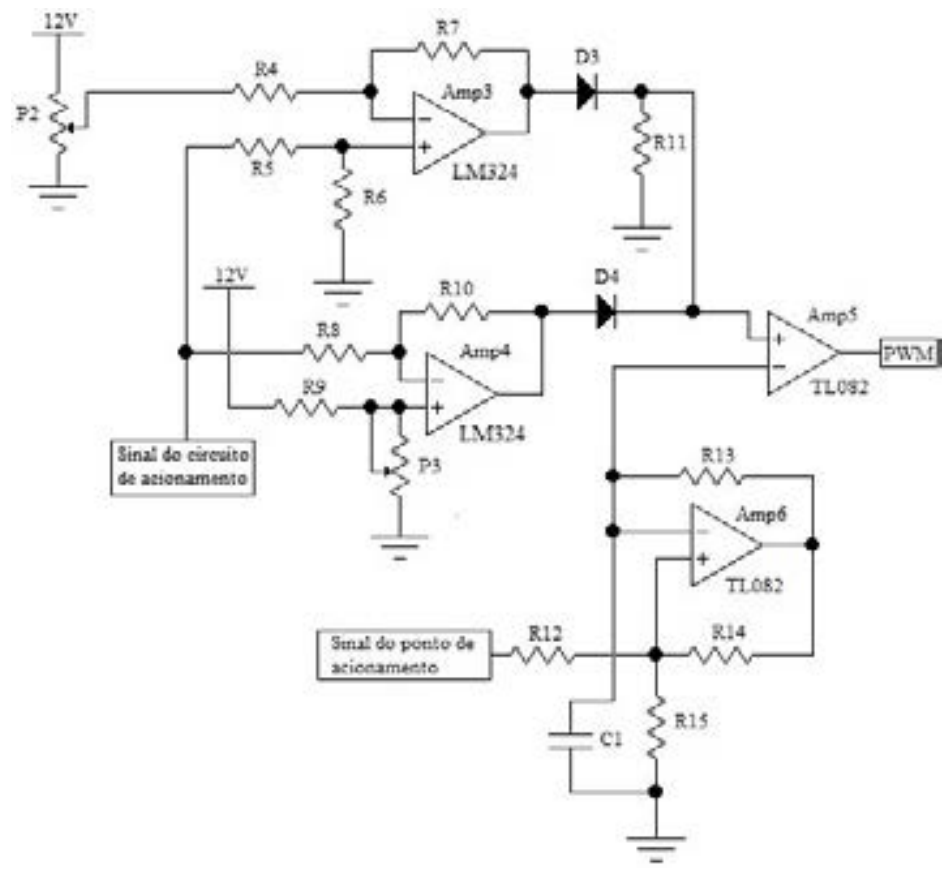

Fonte: (Autores).

Para que as saídas dos amplificadores Amplificador 3 e Amplificador 4 atuem no mesmo ponto sem interferir uma no funcionamento da outra, se fez necessário a aplicação dos diodos $D_{3}$ e $D_{4}$, pois os diodos polarizados conforme ilustrado na figura 3, só permitirão passagem de corrente em um sentido, não permitindo a circulação de corrente de uma saída para outra dos amplificadores. O valor de tensão na entrada não inversora do Amplificador 5 será sempre o sinal da saída que estiver com maior valor. 0 
resistor $\mathrm{R}_{12}$ tem por finalidade garantir que a entrada não inversora do Amplificador 5 nunca fique sem referência caso haja a ausência do sinal do circuito de acionamento. 0 comparador Amplificador 5 é responsável por fazer a comparação do sinal do oscilador com o sinal enviado por um dos amplificadores diferenciais. Quando o sinal enviado por um dos amplificadores tiver o valor de tensão maior que o sinal enviado pelo oscilador, a saída do comparador estará com $12 \mathrm{~V}$, caso contrário a saída estará com 0V, gerando assim o sinal PWM. O circuito de controle faz o monitoramento da posição do eixo de direção através do sensor de posição, conforme ilustrado pela figura 4.

Figura 4 - Circuito do sensor de fim de curso.

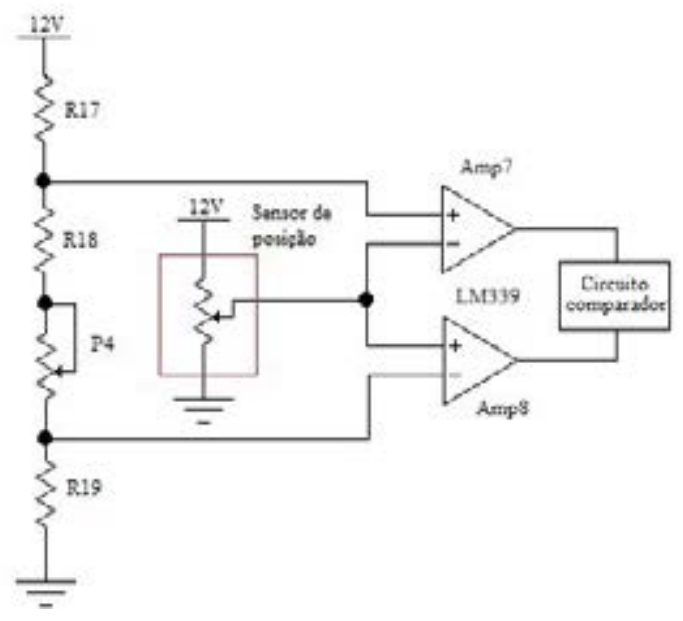

Fonte: (Autores).

O sinal enviado pelo sensor é comparado com dois valores de referência, que são ajustados através de uma malha resistiva divisora de tensão. Quando o valor do sinal enviado pelo sensor de posição for maior que o valor na entrada não inversora do comparador Amplificador 7, a saída do comparador mudará seu valor para OV, fazendo com que circuito driver pare de acionar o circuito de potência. E quando o valor do sinal enviado pelo sensor de posição for menor que o valor na entrada inversora do comparador Amplificador 8, a saída do comparador mudará seu valor para 0V, fazendo com que o circuito driver pare de acionar o circuito de potência. A faixa de ajuste do circuito está dimensionada com valor mínimo de $0,92 \mathrm{~V}$ a $4 \mathrm{~V}$ e valor máximo de $8 \mathrm{~V}$ a $11,07 \mathrm{~V}$, e é 
ajustada pelo potenciômetro $\mathrm{P}_{4^{\prime}}$ que tem seu valor igual a $100 \mathrm{k} \Omega$. Os sinais de saída do circuito de controle são responsáveis por acionar o circuito de potência, e são gerados através da comparação dos sinais enviados pelo circuito do ponto de acionamento, pelo gerador de PWM e pelo controle de fim de curso. O circuito responsável por modelar estes sinais é composto pelos comparadores Amplificador 9, Amplificador 10 e pelas portas $A N D_{1}$ e $A N D_{2}$. Os Amplificador 9 e Amplificador 10 estão inseridos no circuito através de um LM393 e as portas AND ${ }_{1}$ e AND 2 por um CD4081. Isto pode ser visto na Figura 5.

Figura 5 - Circuito comparador que modela os sinais de saída.

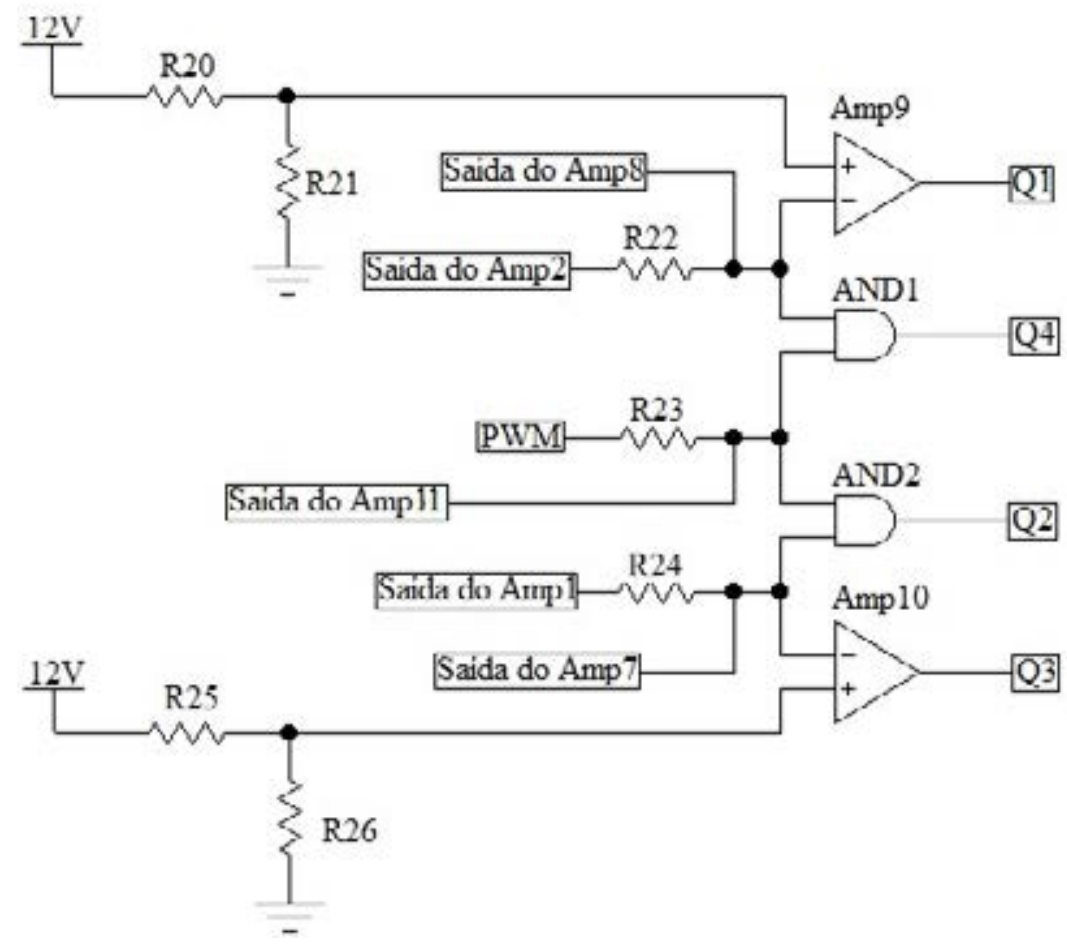

Fonte: (Autores).

Quando o sistema é acionado para um dos sentidos, a saída do Amplificador 1 põe $12 \mathrm{~V}$ na entrada inversora do Amplificador 10 e em uma das entradas da $\mathrm{AND}_{2}$, fazendo com que a saída do Amplificador 10 fique em $0 \mathrm{~V}$ e a saída da $\mathrm{AND}_{2}$ fique dependente apenas do sinal PWM. A entrada inversora do Amplificador 10 e a entrada da porta AND $_{2}$ se manterão em $12 \mathrm{~V}$ enquanto o circuito de fim de curso não mudar de estado a saída do $\mathrm{Amp}_{7}$. E quando o sistema é acionado para o sentido oposto, a saída do Amplificador 2 põe $12 \mathrm{~V}$ na entrada inversora do Amplificador 9 e em uma das entradas da $\mathrm{AND}_{1}$, fazen- 
do com que a saída do Amplificador 9 fique em OV e a saída da AND fique dependente apenas do sinal PWM. A entrada inversora do Amplificador 9 e a entrada da porta AND 1 se manterão em $12 \mathrm{~V}$ enquanto o circuito de fim de curso não mudar de estado a saída do Amplificador 8.

\subsection{Circuito de Potência}

O circuito de potência tem a função de acionar o motor de direção de acordo com as informações enviadas pelo circuito de controle e proteger o motor contra sobrecarga. Através de um controle bidirecional composto por um circuito de ponte $\mathrm{H}$ com mosfet's canal $n$ e canal $p$, é feito o controle do sentido de rotação do motor conforme o diagrama ilustrado na Figura 6.

Figura 6 - Diagrama simplificado do circuito de potência.

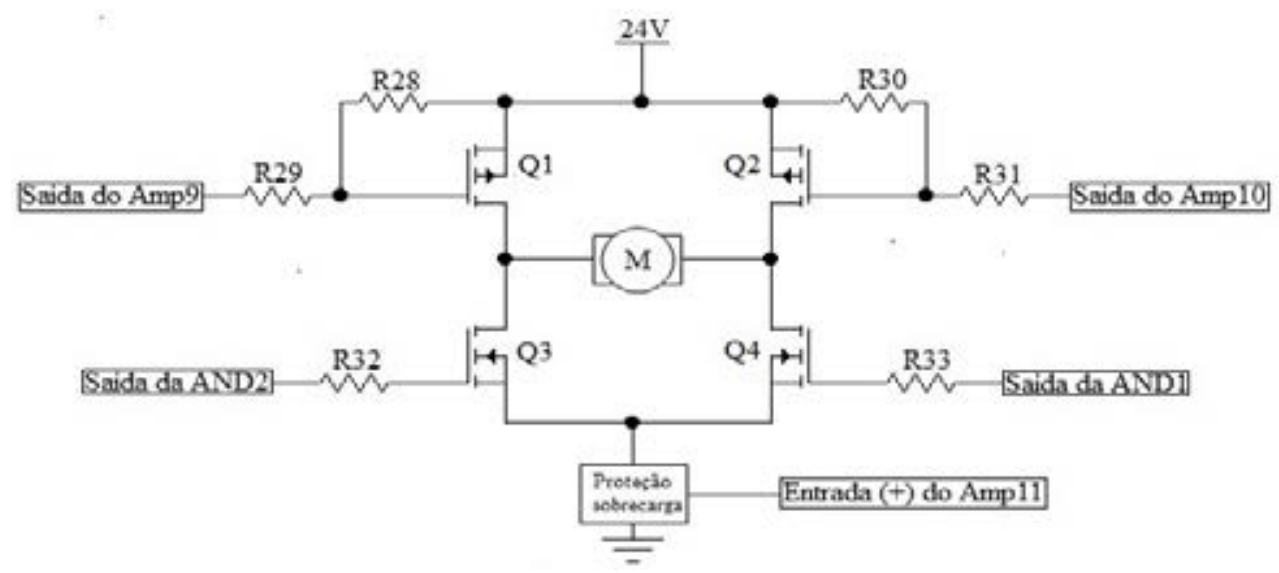

Fonte: (Autores).

$\mathrm{O}$ circuito de potência atua $\mathrm{Q}_{1}$ e $\mathrm{Q}_{4}$ quando o sistema é acionado em um dos sentidos, e $Q_{2}$ e $Q_{3}$ quando o sistema é acionado para o outro sentido. Os sinais de saída do circuito de controle são utilizados para acionar os mosfet's. A saída do Amplificador 9 é utilizada para acionar o $Q_{1}$ e a saída da $A N D_{1}$ é utilizada para acionar o $Q_{4}$, fazendo com que o motor atue em um dos sentidos. Da mesma forma $Q_{2}$ e $Q_{3}$ são acionados, $a$ 
saída do Amplificador 10 é utilizada para acionar o $Q_{2}$ e a saída da $A N D_{2}$ é utilizada para acionar $0 \mathrm{Q}_{3}$, fazendo que o motor atue no sentido oposto. Para o circuito de ponte $\mathrm{H}$, foi escolhido a utilização de quatro mosfet's, dois são canal n IRF3205 e dois são canal p IRF4905, para dimensionar corretamente os componentes foram realizados os cálculos necessários de acordo com os valores propostos pelo projeto, valores estes que são: tensão nominal igual a $24 \mathrm{~V}$ e potência de $300 \mathrm{~W}$. A proteção contra sobrecarga é feita através de um circuito comparador, que efetua a leitura da corrente do motor através de um sensor ACS756, e através dessa leitura é identificado o excesso de corrente em caso de uma sobrecarga e o sistema é bloqueado, conforme mostrado na Figura 7.

Figura 7 - Circuito de proteção contra sobrecarga.

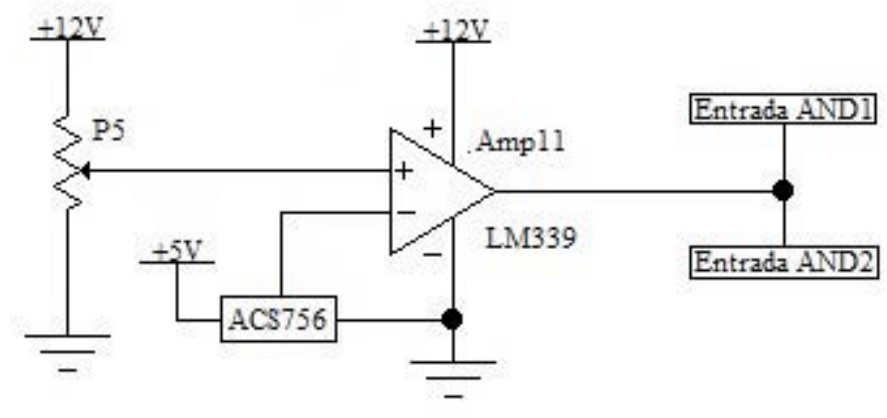

Fonte: (Autores).

O bloqueio é feito quando a corrente do circuito de potência ultrapassar o valor de $I_{\max }$ ou seja, 20A, fazendo com que o sensor ACS756 eleve o nível de tensão na entrada inversora do Amplificador 13. Isso fará com que a saída do Amplificador 13 passe a ter $\mathrm{OV}$, bloqueando assim o funcionamento do sistema através das entradas da $\mathrm{AND}_{1} \mathrm{e}$ $\mathrm{AND}_{2}$.

Através do potenciômetro $P_{5}$, é possível ajustar o valor máximo de corrente que circula pelo circuito de potência, ajustando o valor de tensão na entrada não inversora do Amplificador 11. 


\subsection{Montagem do Protótipo}

O protótipo foi construído para simular e provar a funcionalidade do sistema, e está divido em quatro partes:

- Módulo de comando: trata-se de um sistema mecânico, composto por dois ímãs inversamente polarizados, que acionam o sensor UGN3503. Neste módulo está fixado o circuito de acionamento.

- Módulo de controle: é uma única placa onde estão montados os circuitos de controle e o circuito de potência.

- Motor de direção: O motor utilizado no protótipo, trata-se de um motor de ímãs permanentes, com uma redução mecânica e com um sensor de posição acoplado ao sistema.

- Baterias e chicote elétrico: Foram utilizadas duas baterias seladas de $12 \mathrm{~V}$ associadas em série, para alimentar o sistema com 24V. Foi confeccionado um chicote elétrico, com a finalidade de interligar todos os componentes do sistema. A disposição dos componentes do protótipo é mostrada na Figura 8.

Após a montagem do protótipo, foram realizados todos os ajustes necessários e calibrados antes de o sistema ser acionado. Através das tabelas apresentadas a seguir, estão registrados os valores obtidos de cada ponto dos circuitos.

O circuito de acionamento comportou-se como o esperado, apresentando o ganho muito próximo do valor calculado. O sensor UGN3503 respondeu de forma adequada às influências dos ímãs, comprovando sua sensibilidade à densidade de campo magnético, conforme ilustrado na Tabela 1. 
Figura 8 - Disposição dos componentes do protótipo.

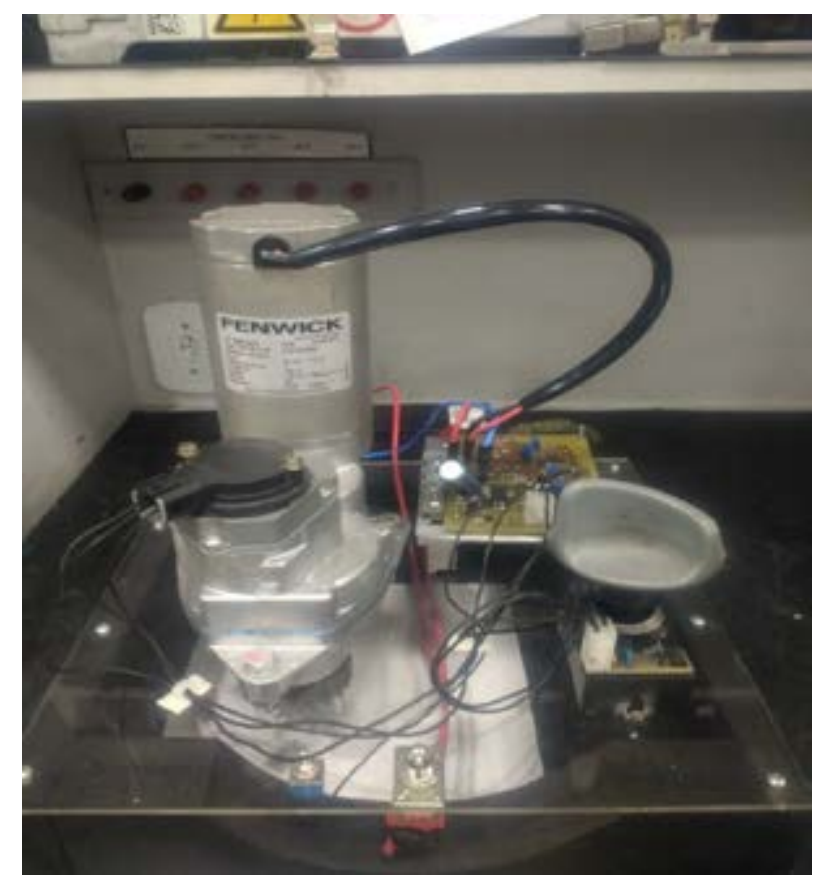

Fonte: (Autores).

Tabela 1 - Comportamento do circuito de acionamento

\begin{tabular}{|l|l|}
\hline Fonte de 5V & $5,12 \mathrm{~V}$ \\
\hline Fonte de 12V & $12,49 \mathrm{~V}$ \\
\hline Sinal do UGN3503 (quando não acionado) & $2,48 \mathrm{~V}$ \\
\hline Sinal do UGN3503 (acionado no sentido horário) & $4,97 \mathrm{~V}$ \\
\hline Sinal do UGN3503 (acionado no sentido anti-horário) & $0,13 \mathrm{~V}$ \\
\hline Sinal de saída (quando não acionado) & $6,01 \mathrm{~V}$ \\
\hline Sinal de saída (acionado no sentido horário) & $11,82 \mathrm{~V}$ \\
\hline Sinal de saída (acionado no sentido anti-horário) & $0,32 \mathrm{~V}$ \\
\hline
\end{tabular}

Fonte: (Autores).

Através dos valores obtidos foi observado que o circuito se comportou de forma 
adequada e satisfatória, com valores experimentais bem próximos dos valores dimensionados no desenvolvimento, com um excelente tempo de resposta. Antes da coleta dos valores apresentado nas Tabelas 2, 3 e 4 todos os ajustes necessários foram realizados através dos potenciômetros $\mathrm{P}_{1^{\prime}}, \mathrm{P}_{2^{\prime}}, \mathrm{P}_{3}$ e $\mathrm{P}_{4^{\prime}}$ para que o circuito funcione com os valores máximos de ajuste de fim de curso, com $100 \%$ de ciclo de trabalho e com maior sensibilidade no acionamento do sistema.

Tabela 2 - Valores obtidos no ponto de acionamento

\begin{tabular}{|l|l|}
\hline $\mathrm{V}_{\text {amp1(-) }}$ & $6,53 \mathrm{~V}$ \\
\hline $\mathrm{V}_{\text {amp2(+) }}$ & $5,61 \mathrm{~V}$ \\
\hline $\begin{array}{l}\text { Sinal de saída do circuito (quando não aciona- } \\
\text { do) }\end{array}$ & $0,35 \mathrm{~V}$ \\
\hline Sinal de saída (acionado no sentido horário) & $11,98 \mathrm{~V}$ \\
\hline $\begin{array}{l}\text { Sinal de saída (acionado no sentido anti-horá- } \\
\text { rio) }\end{array}$ & $11,97 \mathrm{~V}$ \\
\hline
\end{tabular}

Fonte: (Autores).

Tabela 3 - Valores obtidos no circuito gerador de PWM

\begin{tabular}{|l|l|}
\hline $\begin{array}{l}\text { Sinal de saída } \mathrm{Amp}_{3} \text { (quando não aciona- } \\
\text { do) }\end{array}$ & $4,51 \mathrm{~V}$ \\
\hline $\begin{array}{l}\text { Sinal de saída } \mathrm{Amp}_{4} \text { (quando não aciona- } \\
\text { do) }\end{array}$ & $4,53 \mathrm{~V}$ \\
\hline Sinal de saída $\mathrm{Amp}_{3}$ (quando acionado) & $11,74 \mathrm{~V}$ \\
\hline Sinal de saída $\mathrm{Amp}_{4}$ (quando acionado) & $11,76 \mathrm{~V}$ \\
\hline $\mathrm{V}_{\text {amp5(+) }}$ (quando não acionado) & $3,83 \mathrm{~V}$ \\
\hline Frequência do oscilador & $14,48 \mathrm{kHz}$ \\
\hline
\end{tabular}

Fonte: (Autores).

Tabela 4 - Valores obtidos no circuito de fim de curso

\begin{tabular}{|l|l|}
\hline $\mathrm{V}_{\text {amp } 7(+)}$ & $10,59 \mathrm{~V}$ \\
\hline $\mathrm{V}_{\text {amp8(-) }}$ & $1,48 \mathrm{~V}$ \\
\hline Sinal de saída $\mathrm{Amp}_{7}$ (quando acionado) & $0,27 \mathrm{~V}$ \\
\hline Sinal de saída $\mathrm{Amp}_{8}$ (quando acionado) & $0,25 \mathrm{~V}$ \\
\hline
\end{tabular}

Fonte: (Autores). 
O circuito de potência executou o acionamento do motor de acordo com o esperado, de forma controlada, estável e com um excelente tempo de resposta. O calor gerado pelos mosfet's foi dissipado de forma adequada e satisfatória. Os valores obtidos no circuito de potência são apresentados na Tabela 5:

Tensão da bateria $=25,7 \mathrm{~V}$

Tensão no motor (com ciclo de trabalho de $100 \%)=24,3$

Corrente de motor $=6,88 \mathrm{~A}$.

Tabela 5 - Valores obtidos no circuito de proteção contra sobrecarga

\begin{tabular}{|l|l|}
\hline $\mathrm{V}_{\text {amp11(-) }}$ & $3,34 \mathrm{~V}$ \\
\hline $\mathrm{V}_{\text {amp11(+) }}$ (quando não acionado) & $2,51 \mathrm{~V}$ \\
\hline $\mathrm{V}_{\text {amp11(+) }}$ (quando acionado) & $2,76 \mathrm{~V}$ \\
\hline Tensão de alimentação do ACS756 & $5,1 \mathrm{~V}$ \\
\hline
\end{tabular}

Fonte: (Autores).

\section{CONSIDERAÇÕES FINAIS}

O grande diferencial deste projeto é permitir que o operador da máquina consiga manusear a mesma, mesmo na ausência do sistema em decorrência de algum defeito. Isso é possível por se tratar de um sistema assistido e não de um sistema totalmente eletroeletrônico como os existentes no mercado. Pode-se destacar também o fato de que todo o circuito foi tecnicamente desenvolvido de forma simples e com tecnologia de fácil compreensão, o que o torna um dispositivo de fácil manutenção. Isso diminui os custos com a manutenção da máquina, assim como a utilização de um sensor de efeito hall, pois com este tipo de sensor foi eliminado um ponto de desgaste mecânico, que seria causado caso o projeto optasse por um sensor resistivo. 
Com o desenvolvimento de um sistema mecânico e com o avanço de novas tecnologias este sistema pode ser aprimorado e aplicado em diversos outros tipos de máquinas e veículos elétricos, porém novos estudos serão necessários.

Um futuro trabalho a ser realizado seria a implementação deste sistema em uma transpaleteira elétrica, para que seja verificado o comportamento do sistema em condições reais de funcionamento.

\section{REFERÊNCIAS}

ALLEMAND BORGES, Felipe Andrade. Implementação de Sistema de Acionamento para Plataformas Robóticas Móveis com Ênfase em Estimador de Estado de Carga de Bateria. 138 f. Dissertação (Mestrado) - Engenharia Elétrica, Universidade Estadual de Londrina, 2014.

ASHFAQ, Ahmed. Eletrônica de Potência. 1a ed. São Paulo, Editora Prentice Hall, cap.3 e 10, 2000.

BOYLESTAD, Robert. Dispositivos Eletrônicos e Teoria dos Circuitos. 6ạ ed. Rio de Janeiro, Editora LTC, cap.14 e 15, 1999.

DOURADO CARRAH, Israel Franklin. Inversor Monofásico Tipo Ponte Completa com Controle Digital. 187 f. Trabalho de Conclusão de Curso - Engenharia Elétrica, Universidade Federal do Ceará, 2010. 
HART, Daniel W. Eletrônica de Potência - Análise e Projetos de Circuitos. 1a ed. Porto Alegre, Editora AMGH, p.453, 2012.

JUNIOR, Antonio Pertence. Amplificadores Operacionais e Filtros Ativos. 6a ed. Porto Alegre, Editora Bookman, p.51, 2003.

RAMEP. Empilhadeira elétrica: como são e para que servem? Disponível em: < http:// www.ramep.com.br/empilhadeira-eletrica-como-sao-e-para-que-servem/>, Acesso em: 28 abr. 2019, 13:10.

REITER, André. Tipos de direção assistida. Disponível em: <https://www.especialistaemcarros.com.br/single-post/2016/04/26Tipos-de-direção-assistida>, Acesso em: 28 abr. 2019, 11:37.

STILL. Transpaleteiras Elétricas. Disponível em: < https://www.still.com.br/transpaleteiras-eletricas.0.0.html>, Acesso em: 25 mar. 2018, 16:22. 